\title{
Article \\ Using Convolutional Networks and Satellite Imagery to Predict Disease Density in a Developing Country
}

\author{
Rahman Sanya ${ }^{1 *}$ 0000-0002-4631-3980, Gilbert Maiga ${ }^{1}$ and Ernest Mwebaze ${ }^{2}$ \\ 1 School of Computing and IT, Makerere University, Kampala, Uganda; dean@cit.mak.ac.ug \\ 2 AI Lab, Makerere University, Kampala, Uganda; jnakatumba@cis.mak.ac.ug \\ * Correspondence: rsanya@cees.mak.ac.ug
}

\begin{abstract}
Rapid increase in digital data coupled with advances in deep learning algorithms is opening unprecedented opportunities for incorporating multiple data sources for modeling spatial dynamics of human infectious diseases. We used Convolutional Neural Networks (CNN) in conjunction with satellite imagery-based urban housing and socio-economic data to predict disease density in a developing country setting. We explored both single (uni) and multiple input (multimodality) network architectures for this purpose. We achieved maximum test set accuracy of 81.6 per cent using a single input $\mathrm{CNN}$ model built with one convolutional layer and trained using housing image data. However, this fairly good performance was biased in favor of specific disease density classes due to an unbalanced data set despite our use of methods to address the problem. These results suggest $\mathrm{CNN}$ are promising for modeling spatial dynamics of human infectious diseases, especially in a developing country setting. Urban housing signals extracted from satellite imagery seem suitable for this purpose, under the same context.
\end{abstract}

Keywords: Convolutional Networks; satellite imagery; predictive modeling; disease density; urban housing; developing country

\section{Introduction}

Data-intensive computing (big data in concert with advanced machine learning) is projected to play an important role in shaping the future of research in public health generally and computational epidemiology in particular. Several application areas are earmarked in which big data together with deep learning are expected to make significant impact including disease surveillance, medical imaging, medical informatics, bio-informatics, and pervasive sensing [1], [2], and [3]. Current research in this area is however, mostly focused on applications in disease surveillance. For example, Garimella et al. [4] used deep neural networks (DNN) and images posted on social media to infer national health statistics on lifestyle diseases. Ong et al. [5] applied deep recurrent neural networks (DRNN) to predict concentration of particulate matter using textual environmental monitoring data. RNN were also used by Dernoncourt et al. [6] to de-identify patients in textual medical records. Kendra et al. [7] used DNN to analyze and determine discourse on health-related topics using text-based social media content. DNN (skip-gram) were used by Zou et al. [8] on social media data (text) to detect and quantify cases of infectious intestinal diseases. Mobile phone meta-data were used as input data to a Convolutional Neural Network (CNN) [9] and a combination of CNN with Support Vector Machines (SVM) to predict demographic characteristics (age and gender) by Felbo et al. [10]. Lastly, Deep Bayesian Networks (DBN), especially Social Restricted Boltzmann Machines (SRBM) were proposed by Phan et al. [11] for understanding human behavior in health social networks. Communication data consisting of text was used as input to the model.

As can be seen from the example works cited above, research in potential applications of deep learning for disease surveillance is dominated by use of data extracted from social media and other online sources. While an important source that has demonstrated great promise, their applicability in a study may be limited to geographical regions where social media and the Internet are widely used as 
source of health information. For other regions and for population-based risk-related investigations however, alternative sources of digital data must be explored for disease surveillance within the context of digital epidemiology.

In this article we explore opportunities afforded by recent advances in deep learning (DL) for studying disease dynamics using alternative data sources than social media. Specifically, we use Convolutional Neural Networks (CNN) together with satellite imagery-based housing and socio-economic data to predict disease density in a developing country urban setting. Indoor overcrowding (for which we use housing crowding as proxy) and socio-economic well-being are the most important risk factors for our case study disease i.e. Tuberculosis (TB), especially for urban settlements in the study area [12]. TB is a leading cause of death among HIV/AIDS patients in Uganda [13] [14], our study area. The advantage of using DL over conventional machine learning methods is that features used for analysis and prediction are learned automatically from raw input data, thus saving model development complexity. Secondly, DL network architectures especially CNN support simultaneous data input and processing, which is necessary for prediction tasks based on multiple concurrent variables. The contribution of this work is two-fold as follows,

1. We propose a CNN model for predicting disease density. The model takes as input, data from single (or multiple concurrent) source(s) and outputs an estimate of per ca-pita disease density for a geographical region of interest. We instantiated the model by using as input, image data for urban housing and poverty to predict TB in a developing country setting. Our model may be used for infectious disease surveillance and for public health intervention planning.

2. We have established the relative suitability of housing crowding signals extracted from satellite imagery for the purpose of predicting disease density using CNN in a developing country urban setting. This result broadens currently available sources of data for investigating phenomena associated with infectious diseases using machine learning techniques.

The remainder of this paper is structured as follows. Results, discussion, materials and methods are presented in Sections 2, 3, and 4, respectively. We draw conclusions in section 5.

\section{Experimental Results}

The evaluation of our model was done in two phases. First, we evaluated all candidate network architectures for our proposed CNN model on the basis of predictive accuracy. The purpose was to identify architectures that were optimal for the nature and size of our data set from among the single(unimodality) and multiple (multimodality) input architectures. The top three candidates with highest accuracy were then selected for further optimization using a set of hyperparameters to build a robust $\mathrm{CNN}$ model for predicting disease density. Results of evaluating various architectures on prediction accuracy are shown in Table 1 and 2, respectively.

Table 1. Test set accuracy (\%) for single input CNN models.

\begin{tabular}{ccc}
\hline Architecture & Accuracy (poverty data) & Accuracy (housing data) \\
\hline One-layer & 68.4 & $\mathbf{8 1 . 5}$ \\
Two-layer & $\mathbf{8 1 . 6}$ & 78.5 \\
Three-layer & $\mathbf{8 1 . 6}$ & 73.5 \\
Four-layer & $\mathbf{8 1 . 6}$ & 43.9 \\
Five-layer & $\mathbf{8 1 . 6}$ & 43.4 \\
Six-layer & $\mathbf{8 1 . 6}$ & 41.6 \\
\hline
\end{tabular}


Table 2. Test set accuracy (\%) for multi-input CNN models.

\begin{tabular}{cc}
\hline Architecture & Accuracy (poverty + housing data) \\
\hline One-layer & 44.2 \\
Two-layer A & 44.3 \\
Two-layer B & $\mathbf{4 4 . 3}$ \\
Three-layer A & 43.3 \\
Three-layer B & 43.3 \\
Four-layer A & 44.1 \\
Four-layer B & 44.1 \\
Five-layer & 44.3 \\
Six-layer A & 44.3 \\
Six-layer B & 44.3 \\
\hline
\end{tabular}

The results are also summarized using bar plots in Figure 1, 2, and 3, respectively.

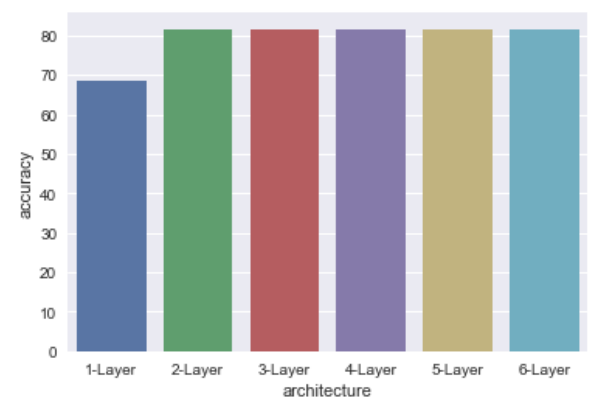

Figure 1. Bar plot showing overall accuracy for single-input CNN models trained on poverty data.

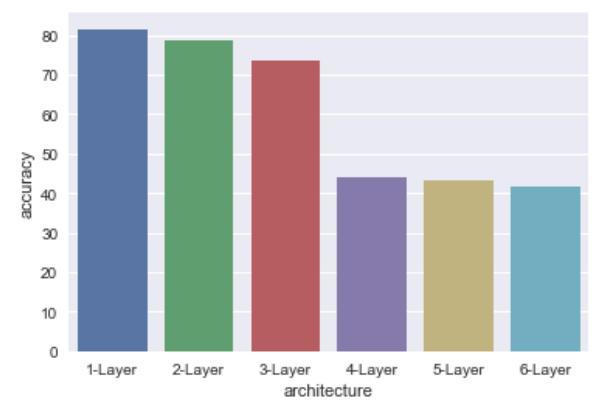

Figure 2. Bar plot showing overall accuracy for single-input CNN models trained on housing data.

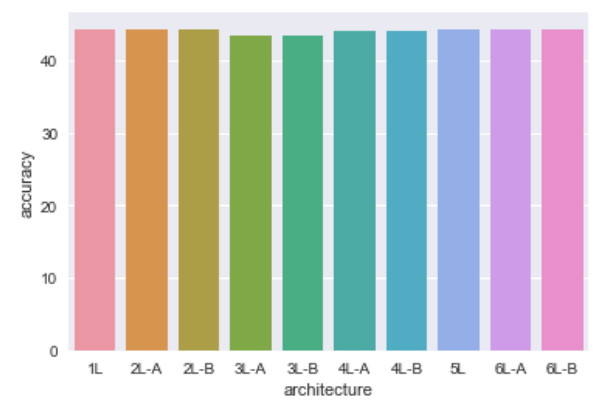

Figure 3. Bar plot showing prediction accuracy for multi-input CNN models trained on concurrent input of housing and poverty data.

The results show that architectures with layer count 2 through 6 trained on poverty data and the 1-layer architecture trained on housing data had the highest predictive accuracy at 81.6 and 81.5 per 
cent, respectively, among the single input architectures (see bold text results in Table 1. On the other hand, the 2-, 5-, and 6-layer architectures performed best all at 44.3 per cent among the multi-input architectures, Table 2. However, accuracy for the best multiple input model at 44.3 per cent was far worse than that for the best single input model at 81.6 per cent. For this reason we selected for further optimization the 1-layer single input model trained on housing data and the 2- and 3-layer models trained on poverty data, based on the principle of parsimony. Our objective here was to squeeze further improvement in predictive accuracy from the three selected architectures.

Hyperparameters we considered for optimizing our three best-performing candidate models include convolution type, kernel size, and filter count. These are some of the important hyperparameters that were not optimized in previous experiments. Further experiments were thus carried out to optimize these trainable parameters on the three candidate models. For convolution type, we adopted the use of depthwise separable convolutions [15] instead of standard convolutions. The former architecture "separates the learning of spatial and channel-wise features" [16]. The result is a lightweight, faster, and more accurate model on the task at hand. Depthwise separable convolutions are also said to learn better representations using small training sets. Three optimization experiments based on depthwise separable convolutions were carried out for each of the three selected models for a total of nine experiments. The results showed zero to negligible improvement in predictive performance for all models, see Table 3.

Table 3. Accuracy (\%) on Standard and Depth-wise separable convolutions.

\begin{tabular}{cccc}
\hline Architecture & Standard & Depthwise & Gain (\%) \\
\hline One-layer & 81.5 & 81.6 & $\mathbf{0 . 1}$ \\
Two-layer & 81.6 & 81.6 & 0 \\
Three-layer & 81.6 & 81.6 & 0 \\
\hline
\end{tabular}

We also wanted to know if larger kernel size and filter count would lead to improvement in prediction accuracy. So we tried out a kernel size of $7 \times 7$ (instead of a $3 \times 3$ one used in previous experiments) and filter count of 128 (instead of 64) on the 1- and 2-layer architectures (selected for their simplicity). Recall that other important hyperparameters such as learning rate, learning rate scheduling, weight decay, etc were already being applied to the previous training processes, so their values were simply maintained throughout these optimization experiments. Three experiments were completed per architecture based on this new hyperparameter configuration for a total of six experiments. Average results are reported in Table 4.

Table 4. Test accuracy (\%) for larger kernel size and filter count for two overall best models.

\begin{tabular}{ccc}
\hline Architecture & Parameter count & Accuracy (\%) \\
\hline One-layer & $4,817,814$ & $\mathbf{8 1 . 6}$ \\
Two-layer & $1,228,182$ & 81.4 \\
\hline
\end{tabular}

The hyperparameter (kernel size and filter count) optimization process resulted in no accuracy improvement for the 1-layer architecture trained on housing data, see Table 4 . In the case of 2-layer architecture trained on poverty data, the test accuracy dropped slightly to 81.4 per cent from 81.6 per cent. We therefore, selected the 1-layer architecture trained on housing data as our overall best model due to its consistent performance and simple design. The performance of this model was further analyzed using additional metrics including confusion matrix (Figure 4), AU-ROC (Figure 5), and precision, recall, and f1-score (Table 5). 


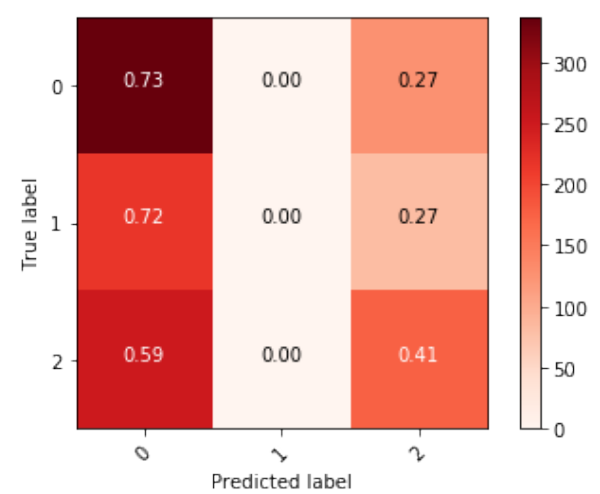

Figure 4. Confusion matrix plot for our overall best model. Key: 0 - high disease density, 1 - low disease density, 2 - moderate disease density.

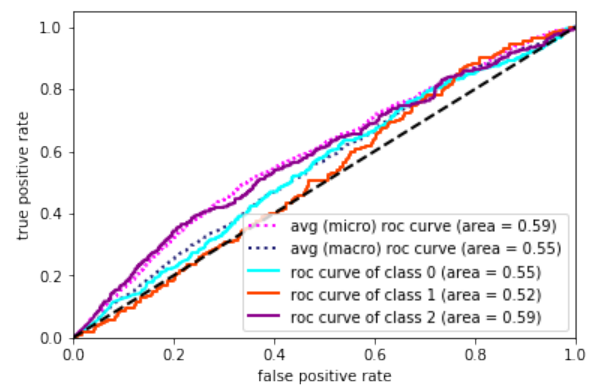

Figure 5. Area under receiver operating characteristic curve (AU-ROC) plot for our overall best model. Key: 0 - high disease density, 1 - low disease density, and 2 - medium disease density.

Table 5. Precision, recall and f1-score values for our overall best model.

\begin{tabular}{cccc}
\hline Disease class & Precision & Recall & F1-Score \\
\hline High disease density & 0.83 & 0.58 & 0.69 \\
Moderate disease density & 0.68 & 0.84 & 0.75 \\
Low disease density & 0.87 & 0.78 & 0.83 \\
\hline
\end{tabular}

As indicated earlier we employed visualization to scrutinize and interpret (explain) classification decisions of our model i.e., we wanted to know what features were being learned by the model and used for classifying a geographical region captured in image data as a place likely to have high, moderate, or low disease density. For this purpose we employed gradient-weighted class activation mapping (grad-CAM) [17], [18], a technique for visualizing CNN predictions based on features learned by the deepest convolutional (or pooling) layer in a CNN pipeline. The visualizations presented in Figures 6 through 11 are based on our overall best model i.e., the 1-layer single input model trained on housing image data. Recall that 1-layer here means there is only one convolutional layer in the pipeline and hence, visualizations are based on this one convolutional layer. Our visualization efforts posted mixed results in that some features learned by the model were visualize-able while others were not. For example, in the case of high disease density class, features learned from some images were visually perceptible while they were not in others as seen in Figures 6 and 7. A similar situation manifests when visualizing features of the moderate and low disease density classes, as seen in Figures 8, 9, 10, and 11, respectively. In each of the figures, column 1 (from left) is the input image. Vanilla, guided, and relu (columns 2,3,4) are different options for modifying the backpropagation algorithm i.e., no modification, Guided Backpropagation (only propagate positive gradients for positive activations) [19], and ReLU (only propagate positive gradients), respectively. The latter two options appear to give us better results than the former. 

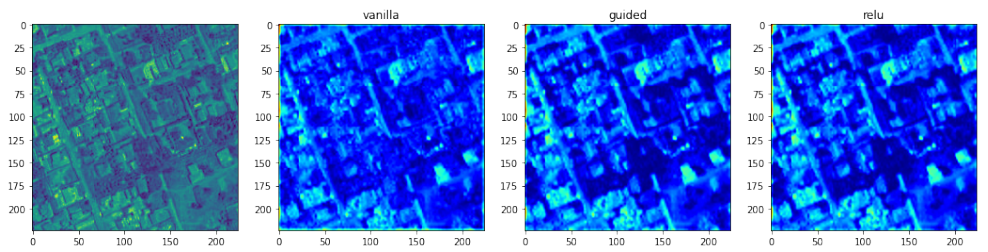

Figure 6. Visualizing features used in predicting class: high disease density. Case where learned features were visually perceptible.
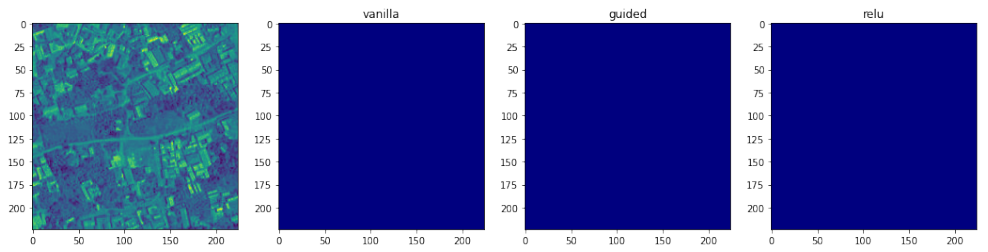

Figure 7. Visualizing features used in predicting class: high disease density. Case where learned features were visually imperceptible.
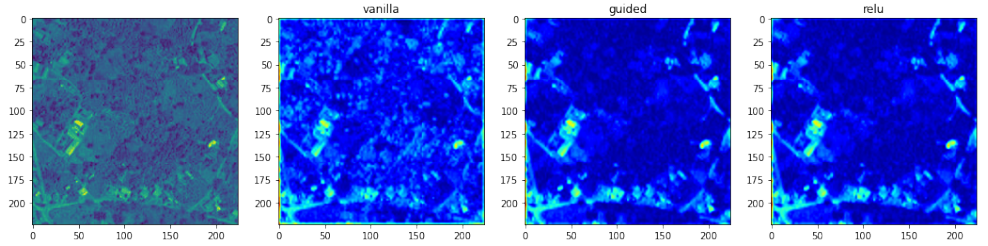

Figure 8. Visualizing features used in predicting class: moderate disease density. Case where learned features were visually perceptible.
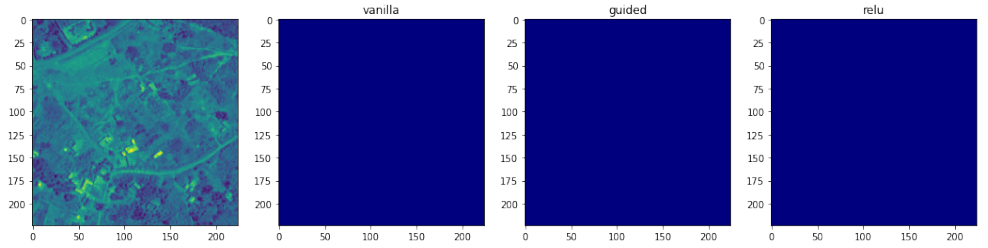

Figure 9. Visualizing features used in predicting class: moderate disease density. Case where learned features were visually imperceptible.
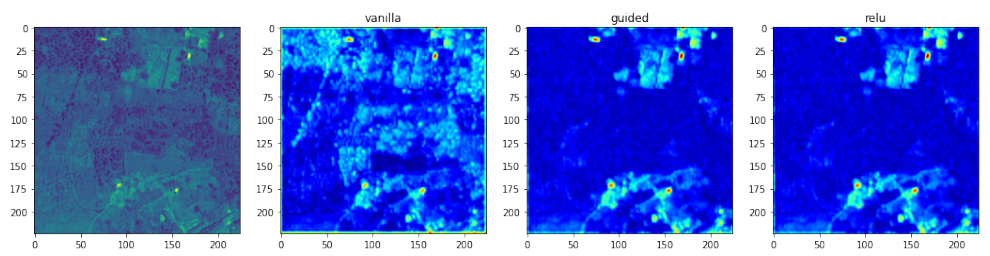

Figure 10. Visualizing features used in predicting class: low disease density. Case where learned features were visually perceptible. 


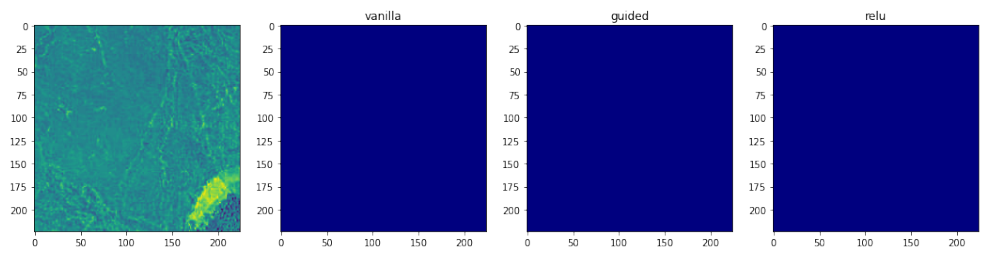

Figure 11. Visualizing features used in predicting class: low disease density. Case where learned features were visually imperceptible.

In general, it is clear from our visualizations of class activation maps (columns 2, 3, and 4) in Figures 6, 8, and 10 that our model is learning (and using), more so correctly, features associated with housing to make classification decisions.

\section{Discussion}

The goal of this work was to develop a CNN model for predicting disease density. The primary model objective was therefore, prediction accuracy followed by transparency. To attain this goal we needed to,

- Identify a CNN network architecture that was optimal for the nature and size of data set we had,

- Tune a set of hyperparameters to build a model with high predictive accuracy and ability to generalize well on data previously not seen by the model.

For the first task we evaluated an architecture search space consisting of 10, 2-branch multi-input and 6, single-input architectures up to 6 layers deep. We found that a 1-layer single-input architecture constructed with a depthwise separable convolution is most optimal when trained on housing satellite image data. A model built using this architecture gave the highest test set accuracy of 81.6 per cent using hyperparameter values and/or methods specified in Table 12, in addition to using a kernel size of $3 \times 3$ and filter count of 64 . The model also performed well in terms of predicting individual classes (Figure 4), exactness (precision), and completeness (recall), Table 5. The accuracy attained by our model, more over with a small training set, falls within range of current state-of-the-art results obtained elsewhere on similar data sets. For example, [20], [21], [22], [23], and [24] all worked with satellite imagery data and $\mathrm{CNN}$ (or other deep learning framework) for different classification problems and obtained accuracy between 75 and 97 per cent (Table 6).

Table 6. Classification accuracy on different satellite scene image data sets.

\begin{tabular}{cccccc}
\hline Study & Predicting & Algorithm & Image count & Training method & Accuracy (\%) \\
\hline Current work & Disease density & CNN & 12,070 & Fresh model & 81.6 \\
Sanya et al. [20] & Housing density & VGGNet16 & 31,113 & Transfer learning & 79.5 \\
Albert et al. [21] & Urban environments & ResNet-50 & 140,000 & Transfer learning & 83.0 \\
Cheng et al. [22] & Object class & VGGNet16 & 31,500 & Transfer learning & 90.3 \\
Jean et al. [23] & Poverty & VGGNet & 1,411 & Transfer learning & 75.0 \\
Basu et al. [24] & Object class & DBN & 500,000 & Fresh model & 97.9 \\
\hline
\end{tabular}

These seemingly low accuracy rates compared to other well-researched image data sets such as Imagenet [25] attests to the difficulty of the task when using remotely sensed imagery to classify scenes using high-level, subjective semantic concepts given that this field of research is still in its early stages. The difficulty arises from the fact that scene images are a complex phenomenon due to high inter-class similarity and intra-class diversity, and using high-level subjective descriptions for classification makes it even a harder task for deep learning algorithms.

An additional requirement for our model was that it should be possible for humans to interpret decision processes of the model (correct and incorrect decisions) in terms of semantic features of input data. Since the overall best model was found to be the 1-layer architecture trained on housing data, this 
means that we must be able to explain the model's decisions in terms of housing information. Indeed, it was found that the model was learning the correct features i.e., those associated with 'buildings' in the input images to make decisions for disease density classification. However, visualization results were a mixed bag of success in that it was not possible to 'visually explain' all learned features since some were not perceptible to the visualization algorithm. This is understandable since it is shown in [18] that visualizations tend to be poor at shallow convolutional layers but get progressively better at deeper ones. The reason suggested for this is that deeper convolutional layers learn higher-level semantic features and also keep spatial information, while shallower layers have small receptive fields and focus on local features used by next layers.

With respect to predicting epidemiological phenomena, a number of other studies have used artificial neural networks (ANN). For example, Moustris et al. [27] and Capinha et al. [28] applied Multi-layer Perceptrons (MLP) [26] to predict asthma admissions and habitat suitability for malaria vectors, respectively, using environmental and related variables. In an earlier study, Kiang et al. [29] used ANN to predict malaria transmission dependency on four environmental factors. The work most related to ours is that of Rajabi et al. [30] who used Radial Basis Functional Link Nets (RBFLN) [31] with environmental variables to predict Visceral Leishmaniasis distribution patterns. They posted 92 per cent accuracy, which is better than what is scored in the current study (81.6 per cent). This however, is expected given that the former study used a large number of risk variables (7) in their prediction model compared to what is used in the present study (2 risk variables for the case study disease [12]), see Table 7.

Table 7. Classification accuracy (\%) for ANN models on epidemic prediction tasks.

\begin{tabular}{ccccc}
\hline Study & Prediction task & Algorithm & No. variables & Accuracy (\%) \\
\hline Current work & Disease density & CNN & 2 & 81.6 \\
Rajabi et al. [30] & Epidemic pattern & RBFLN & 7 & 92.0 \\
Moustris et al. [27] & Hospital admissions & MLP-TLRN & 7 & IA=0.838 \\
Capinha et al. [28] & Vector habitat suitability & MLP-ANN & 5 & kappa $=0.42$ \\
Kiang et al. [29] & Transmission dependency & ANN & 4 & 62.9 \\
\hline
\end{tabular}

\section{Materials and Methods}

\subsection{Data set}

\subsubsection{Population data}

The population data we used is a public data set provided by Facebook Connectivity Lab and Center for International Earth Science Information Network (FB-CIESIN) [32]. The data estimates population distribution per $30 \mathrm{~m} \times 30 \mathrm{~m}$ grid based on recent census data and high resolution $(0.5 \mathrm{~m})$ satellite imagery. We up-sampled the data to $250 \mathrm{~m} \times 250 \mathrm{~m}$ grid to be consistent with our spatial unit of analysis. The population data was used to dis-aggregate epidemic data from a larger geospatial unit (sub-county) to $250 \mathrm{~m} \times 250 \mathrm{~m}$ grid using the method described in Section 4.2.2. Raster image for population distribution covering the study area for year 2015 is shown in Figure 12. 


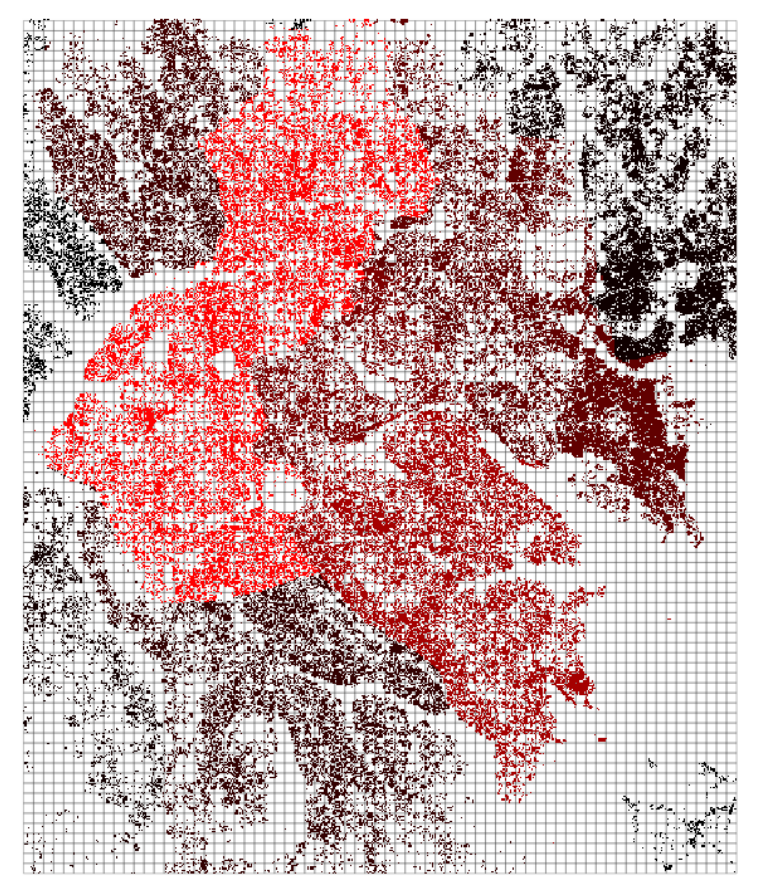

Figure 12. Population distribution data for year 2015 covering the study area.

\subsubsection{Socio-economic data}

The socio-economic data [33], a publicly available data set provides estimates of the proportion of poor people living in a $1 \mathrm{~km} \times 1 \mathrm{~km}$ grid in Uganda for the year 2015. Measurement of well-being is based on a multi-dimensional poverty index (MPI), which adopts a broad definition of well-being proposed by Alkire et al. [34]. The data in raster file format (geotiTIFF) was down-sampled from $1 \mathrm{~km} \mathrm{x}$ $1 \mathrm{~km}$ to $250 \mathrm{~m} \times 250 \mathrm{~m}$ grid so as to be consistent with our spatial unit of analysis. The data was used as input for training our CNN models. The original data set was downloaded from the WorldPop website http://www.worldpop.org/.

\subsubsection{Housing data}

The housing data, also available in the public domain, provides indication of housing or building crowding in a $250 \mathrm{~m} \times 250 \mathrm{~m}$ grid. This data consists of satellite imagery extracted from Google Static Maps API https://maps.googleapis.com/maps/api/staticmap, using a method described in [20]. The housing data was used as input for training our CNN models. Example images taken from our housing data set are shown in Figure 13. 


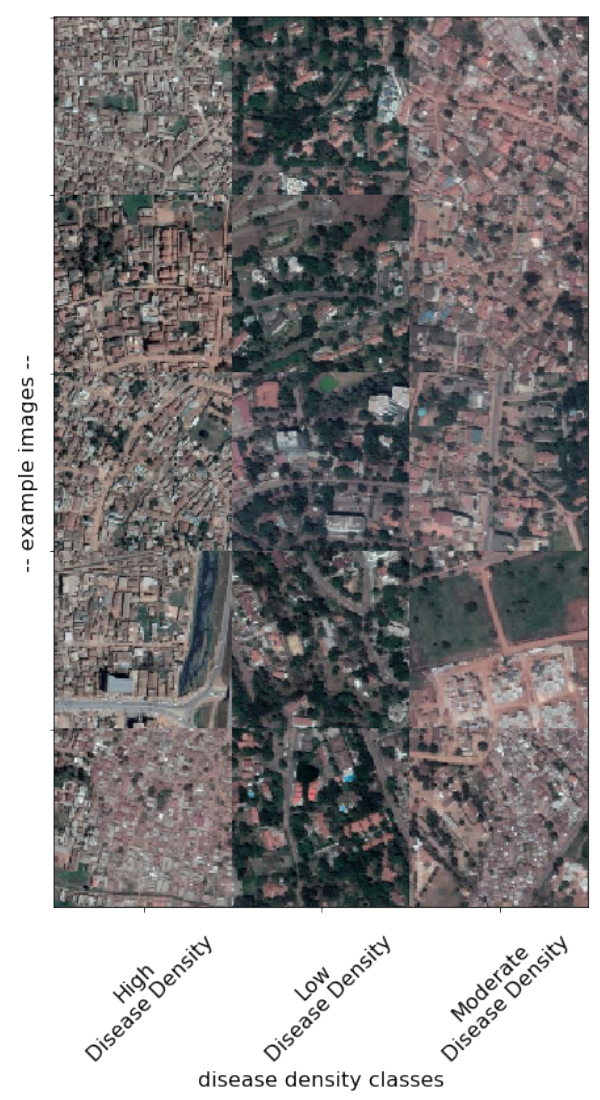

Figure 13. Example housing image data for study area extracted from Google Static maps API.

\subsubsection{Epidemic data}

The disease used as case study in this work is Tuberculosis (TB), a high burden infectious disease and a leading cause of death among HIV/AIDS patients in Uganda [13] [14]. The data consists of monthly disease case counts for the year 2015 reported at sub-county level. It was acquired from the Health Management Information System version 2 (HMIS2), Ministry of Health, Uganda. The epidemic data was disaggregated from sub-county level counts to $250 \mathrm{~m} \times 250 \mathrm{~m}$ grid estimates using a method described in Section 4.2.2.

\subsection{Methods}

\subsubsection{Study area}

The study area includes two large urban areas namely Kampala (the capital city) and Masaka in Uganda. Uganda lies between 10 29' South and 40 12' North latitude, 29034 East and 350 0' East longitude. These two urban centers are highly urbanized, have large populations, and report high infectious disease occurrence (especially TB and HIV / AIDS), which makes them suitable for the kind of analyses in this work. The spatial unit of analysis is a grid (cell) of size $250 \mathrm{~m} \times 250 \mathrm{~m}$ (approx. 224 x 224 pixels), chosen to comply with satellite image extraction constraints and for easy analysis and interpretation of disease density prediction results.

\subsubsection{Disaggregating and discretizing epidemic data}

Since our spatial unit of analysis is a $250 \mathrm{~m} \times 250 \mathrm{~m}$ grid (cell), we required to scale our epidemic data to this unit to ensure consistency across all data sets. Therefore, to disaggregate the data from large geospatial unit counts (sub-county) to $250 \mathrm{~m} \times 250 \mathrm{~m}$ grid count, we took advantage of the relationship between population contact-density structure $c$ and infection transmission. Although findings on the 
exact shape of the mathematical function describing this relationship is inconclusive, we assumed a linear one as suggested in [35] and [36], Equation (1),

$$
c=a\left(\frac{N}{A}\right)
$$

where $a$ is fitting parameter, $N$ is population size, and $A$ is area size. We further tested this relationship by analyzing observational epidemic data with census population data at low spatial resolution and found significant positive correlation $(p=0.000)$ and average $r^{2}=0.59$. Before disaggregating the epidemic data to grid estimates, we first weighted each grid by its estimated population using Equation (2),

$$
w_{i, j}=\frac{\rho_{i, j}}{\sum_{s} \rho}
$$

where $w$ is weight assigned to a grid located at the intersection of row $i$ and column $j$, and $\rho$ is its population size. We now estimate disease count $d$ at this grid using Equation (3) [37],

$$
d_{i, j}=D_{s} * w
$$

where $D_{s}$ is disease count at the larger spatial unit (sub-county) in which grid at intersection $i, j$ lies and $w$ is the grid's weight. Having disaggregated the epidemic data to grid scale, we needed to create disease density classes out of the data to make it a classification task. To do this we binned the normalized data such that each grid assumed a class depending on which bin its disease case estimate falls in. By following the procedure in [38] for binning population data, we created a matrix $C$ where an entry $C^{i, j}=0$ if $0.66<d^{i, j}=<1.00,2$ if $0.33<d^{i, j}=<0.66$, and 1 if $0.00=<d^{i}, j=<0.33$ where $d^{i, j}$ is normalized disease estimate. Table 8 provides details of disease density classes used in the present work. A summary of data sets used in this research is provided in Table 9.

Table 8. Disease density classes used in the present work.

\begin{tabular}{cccc}
\hline Value range & Class & Class label & Sample count \\
\hline $0.67-1.00$ & 0 & High disease density & 3,686 \\
$0.34-0.66$ & 2 & Moderate disease density & 1,898 \\
$0.00-0.33$ & 1 & Low disease density & 6,486 \\
\hline
\end{tabular}

Table 9. Summary of data sets used in the present work.

\begin{tabular}{ccc}
\hline Data set & Use & Source \\
\hline Poverty & Input to model & WorldPop \\
Housing & Input to model & Google Static Maps API \\
Epidemic & Disease class label & HMIS2 \\
\hline
\end{tabular}

All input data was pre-processed into appropriate feature vectors and normalized to $(0,1)$ value range by min-max scaling (Equation 4 ) or, $(1,1)$ by standardizing (Equation 5),

$$
\begin{aligned}
X_{\text {norm }} & =\frac{x-x_{\text {min }}}{x_{\max }-x_{\text {min }}} \\
z & =\frac{x-\mu}{\sigma}
\end{aligned}
$$

\subsubsection{Model architecture and configuration}

The goal of this work was to predict disease density using both uni- and multimodality representation learning. To achieve this goal we trained a CNN model that takes as input, housing 
and/or socio-economic imagery data and outputs disease density class for a given geographical region of interest.

Let $D$ be a grid of disease count values for a given geographical area at time $t, C$ a grid of target disease class values, $H$ a grid of satellite images (depicting housing crowding), and $E$ a grid of socio-economic indicator values. Thus, for every disease data value $d^{i, j}$ and target class value $c^{i, j}$ there is an associated satellite image $h^{i, j}$ and socio-economic indicator value $e^{i, j}$. We formulated the learning task as estimating function $g$ using Equation (6) for a multi-input, single-output prediction problem,

$$
c^{i, j}=g\left(h^{i, j}, e^{i, j}\right)
$$

where the multimodality (multi-input) representation $c^{i, j}$ is computed using function $g$ based on unimodality (single-input) representations $h^{i, j}, e^{i, j}$ (see [39] for a survey on multimodality machine learning). For CNN models based on overall unimodality architecture, representation learning was done using only one data source (either housing or socio-economic data) as shown in Equations 7 and 8 , respectively.

$$
\begin{aligned}
& c^{i, j}=g\left(h^{i, j}\right) \\
& c^{i, j}=g\left(e^{i, j}\right)
\end{aligned}
$$

In this work we used a CNN to estimate the function $g$ since the mapping from input data to disease density estimate is non-linear, noisy, and dependent on semantic content of input data. An overview of the one-layer unimodality (single-input) and one-layer multimodality (multi-input) CNN network architectures are shown in Figures 14 and 15, respectively.

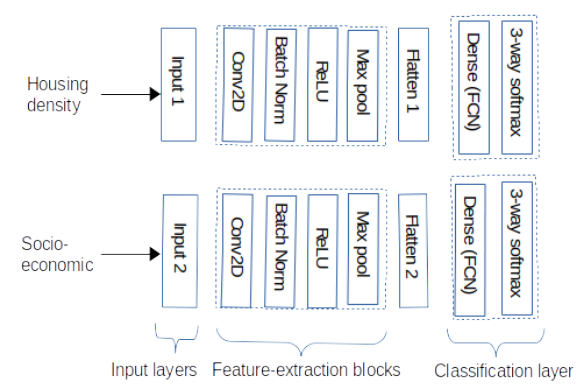

Figure 14. Our one-layer unimodality $\mathrm{CNN}$ architecture for predicting disease density.

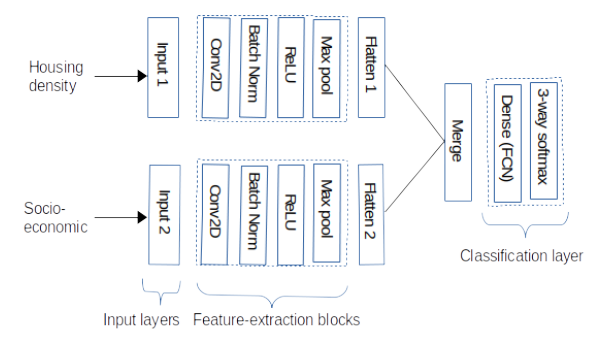

Figure 15. Our one-layer multimodality $\mathrm{CNN}$ architecture for predicting disease density.

The core element of each network architecture is a feature extraction (FE) block consisting of the following sequence of four different types of layers: a convolution (conv2D), batch normalization, activation (ReLU), and max pooling. For a multimodality network, the two input branches are merged (by concatenation) before a classification layer consisting of a single dense layer with a 3-way softmax activation function is applied. 
Table 12. Hyperparameter values or methods used to set them in our experiments.

\begin{tabular}{cc}
\hline Hyperparameter & Value or method \\
\hline Activation function (convolution layer) & ReLU \\
Activation function (classification layer) & Softmax \\
Optimization & SGD (Adadelta) \\
Loss function & Multi-class cross-entropy \\
Learning rate & 0.1 (halved every 10 epochs) \\
Batch normalization & (momentum $=0.9$ ) \\
Weight initialization & Random \\
Weight decay type (kernel regularizer) & L2 $(0.01)$ \\
Amount of weight decay & 0.0002 \\
Drop out & Not used \\
Image data augmentation & Yes \\
Bias initialization & Zeros \\
Mini-batch size & 32 \\
Number of epochs & 30 \\
\hline
\end{tabular}

An architecture search space consisting of ten multimodality (Table 10) and six unimodality architectures (Table 11) for a total of sixteen, up to six layers deep, were evaluated. The goal was to identify a CNN architecture that was optimal for the nature and size of our data set.

Table 10. Architecture search space for multimodality CNN model (FE - feature extraction blocks).

\begin{tabular}{cccc}
\hline Architecture & FE before merging & FE after merging & Parameter count \\
\hline One-layer & 1 & 0 & $4,820,739$ \\
Two-layer A & 1 & 1 & 679,875 \\
Two-layer B & 2 & 0 & $1,243,395$ \\
Three-layer A & 2 & 1 & 151,395 \\
Three-layer B & 3 & 0 & 789,507 \\
Four-layer A & 2 & 2 & 132,387 \\
Four-layer B & 4 & 0 & $1,079,811$ \\
Five-layer & 5 & 0 & $3,291,651$ \\
Six-layer A & 3 & 3 & 357,411 \\
Six-layer B & 6 & 0 & $12,639,747$ \\
\hline
\end{tabular}

Table 11. Architecture search space for unimodality CNN model.

\begin{tabular}{ccc}
\hline Architecture & Feature Extraction blocks (FE) & Parameter count \\
\hline One-layer & 1 & $2,410,371$ \\
Two-layer & 2 & 641,091 \\
Three-layer & 3 & 226,563 \\
Four-layer & 4 & 150,723 \\
Five-layer & 5 & 159,555 \\
Six-layer & 6 & 188,931 \\
\hline
\end{tabular}

Hyperparameter settings used for training our models are shown in Table 12. The hyperparameter values were either obtained directly from the literature based on work using similar data set e.g., for learning rate and learning rate scheduling [21] or recommended best practices e.g., for weight initialization [40]. Settings specific to convolutional layers included a $3 \times 3$ (and $7 \times 7$ ) filter size, filter count of 64 (and 128), stride 1 x 1, and zero-padding as "same".

\subsubsection{Model training}

In this work, we adopted a strategy that involves developing a new model from scratch (as opposed to using transfer learning based on pre-trained models) for several reasons. For example, this 
strategy allows for exploring of new network architectures that best fit the problem at hand in order to arrive at a robust model, which would not have been possible with transfer learning. Besides, there were no pre-trained multimodality CNN models in the public domain known to us by the time this study was conducted. Despite its advantages however, this strategy is laborious, time-consuming, and compute-intensive.

In developing and implementing each proposed $\mathrm{CNN}$ network architecture an iterative process was followed consisting of the following phases of activities: designing the architecture, training a model based on it, and analyzing/validating the model, Figure 16. Once a satisfactory model was realized, it was trained on a combined data set of training and validation sets before evaluating on the test set. We applied the same regularization methods and other hyperparameter values to ensure uniformity across all architectures.

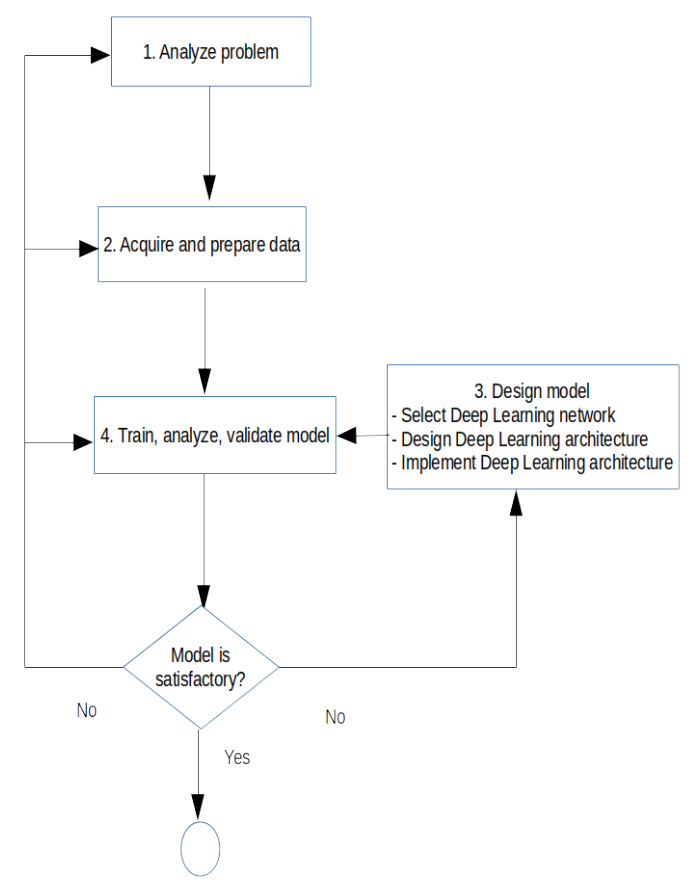

Figure 16. Overview of the iterative process we used to build a CNN model for predicting disease density.

\subsubsection{Experimental setup}

Our experiments were implemented in Keras [41], a popular open source deep learning framework using TensorFlow [42] back-end. Specifically, the Keras Functional API was used to implement the multimodality $\mathrm{CNN}$ model. Common regularization techniques namely data augmentation were utilized during training to improve model generalization capability i.e. to overcome overfitting. Data augmentation techniques used included random rotation (15 degrees maximum either direction), shearing (up to 0.1 radians), zooming (0.2), horizontal and vertical flipping. See Table 12 for additional experimental settings. Images were also shuffled during training to avoid influencing the model by order in which the images were being fed. Input image dimension was $224 \times 224 \times 3$ composed of red, green, and blue (RGB) spectral bands for housing data and $224 \times 224 \times 1$ for poverty data. We used a ratio of 80:20 per cent to randomly split the data into a train-test set. Since our training data is unbalanced in respect of some classes (i.e. the ratio is $1.75: 1: 3.4$ ), we weighted the classes to give higher weight to under represented ones. We completed three train-test cycles per architecture during each phase of evaluating candidate models, for a total of 63 experiments (i.e., $16 \times 3,3 \times 3$, and $2 \times 3$ ), in order to arrive at an overall best model out of a search space consisting of 16 potential architectures. 
Early stopping was implemented to stop model training whenever the validation accuracy did not improve after 10 epochs. Table 13 shows size of our training and testing data sets.

Table 13. Training and test data set size.

\begin{tabular}{cc}
\hline Dataset & Size \\
\hline Training set & 12,070 \\
Test set & 1,190 \\
Total & $\mathbf{1 3 , 2 6 0}$ \\
\hline
\end{tabular}

\subsubsection{Model evaluation}

We used five standard evaluation metrics commonly used in machine learning research to evaluate the performance of our disease density prediction model. These include confusion matrix, overall accuracy, area under receiver operating characteristic curve (AUC/ROC), precision, recall, and F1-score. The decision to use multiple metrics is based on the reasoning that subjecting the resultant model to many different validation tests would give us a truly robust and highly generalizeable model. We employed visualization to gain further insight into and, explain model predictions using semantic features of input data i.e., housing and socio-economic image data. Our visualizations are based on a category of techniques called Class Activation Maps (CAM) which involve producing heatmap of class activations over input images. Unlike saliency maps [43] [44] that use gradients with output of the classification (dense) layer, the more general grad-CAM [17] [18] uses gradients with output of the last convolutional (or pooling) layer so as to utilize spatial information that gets completely lost in dense (classification) layers.

\section{Conclusions}

In this work we have considered the question of whether or not a CNN model can reasonably predict disease density from image-based urban housing and socio-economic data, especially in context of a developing country. Our experimental results show that $\mathrm{CNN}$ are promising for this task. In the current study, a CNN model built with a 1-layer depthwise separable convolution and appropriately optimized with a set of hyperparameters has the best predictive accuracy given our relatively small training data. Our model also generalizes fairly well to previously unseen data. Building signals extracted from satellite imagery was also found to be suitable input data source for predicting disease density using CNN. Despite the promising results achieved with a shallow architecture however, insufficient training data was found to be a limiting factor on the predictive performance of deeper and multi-input architectures. It would therefore, be interesting to know if and how predictive accuracy of multi-input architectures scales with larger training data. It is also worth noting that the current results were achieved with two risk variables for our case study disease. A multi-input CNN model for predicting disease density based on a larger number of risk variables would be an interesting research direction to pursue.

Author Contributions: Conceptualization, Rahman Sanya and Ernest Mwebaze; Data curation, Rahman Sanya; Investigation, Rahman Sanya; Methodology, Rahman Sanya; Resources, Rahman Sanya; Supervision, Gilbert Maiga; Validation, Rahman Sanya; Writing - original draft, Rahman Sanya; Writing - review \& editing, Gilbert Maiga and Ernest Mwebaze.

Funding: This research received no external funding.

Conflicts of Interest: The authors declare no conflict of interest.

\section{References}

1. Salathe, M. Digital epidemiology: what is it, and where is it going? Life Sciences, Society and Policy 2018, 14(1), doi:10.1186/s40504-017-0065-7. 
2. Ravi, D., Wong, C., Deligianni, F., Berthelot, M., Andreu- Perez, J., Lo, B., and Guang-Zhong. Deep learning for health informatics. IEEE Journal of Biomedical and Health Informatics 2017, 21(1).

3. Marathe, M. and Vullikanti, A. K. S. Computational epidemiology. Communications of the ACM 2013, 58(7), $88-96$.

4. Garimella, V. R.K., Alfayad, A. and Weber, I. Social media image analysis for public health. CoRR 2015, abs/1512.04476.

5. Ong, B. T., Sugiura, K., and Zettsu, K. Dynamically pre-trained deep recurrent neural networks using environmental monitoring data for predicting pm2.5. Neural Computing and Applications 2016, 27, 1553-1566.

6. Dernoncourt, F., Lee, J. Y., Uzuner, Ö. and Szolovits, P. De-identification of patient notes with recurrent neural networks. CoRR 2016, abs/1606.03475.

7. Kendra, R. L., Karki, S., Eickholt, J. L. and Gandy, L. Characterizing the discussion of antibiotics in the twittersphere: What is the bigger picture? J Med Internet Res Jun 2015, 17(6):e154.

8. Zou, B., Lampos, V., Gorton, R. and Cox, I. J. On infectious intestinal disease surveillance using social media content. In Proceedings of the 6th International Conference on Digital Health Conference, DH '16; ACM: New York, NY, USA, ACM, 2016; pp. 157-161.

9. Lecun, Y., Leon, B., Yoshua, B., Patrick, H. Gradient-based learning applied to document recognition. In Proceedings of the IEEE 1998, 2278-2324.

10. Felbo, B., Sundsøy, P. R., Pentland, A., Lehmann, S. and de Montjoye, Y. Using deep learning to predict demographics from mobile phone metadata. CoRR 2015, abs/1511.06660.

11. Phan, N., Dou, D., Piniewski, B. and Kil, D. Social restricted boltzmann machine: Human behavior prediction in health social networks. In 2015 IEEE/ACM International Conference on Advances in Social Networks Analysis and Mining (ASONAM) Aug 2015, 424-431.

12. Kirenga, B. J., Ssengooba, W., Muwonge, C., Nakiyingi, L., Kyaligonza, S., Kasozi, S., Mugabe, F., Boeree, M., Joloba, M., Okwera, A. Tuberculosis risk factors among tuberculosis patients in Kampala, Uganda: Implications for tuberculosis control. BMC Public Health 2015, 15(13), 1-7. doi:10.1186/s12889-015- 1376-3.

13. World Health Organization. Global tuberculosis report 2017. Technical report, 2017; World Health Organization.

14. UNAIDS. Global report: UNAIDS report on the global aids epidemic. Technical report 2013; United Nations.

15. Chollet, F. Xception: Deep learning with depthwise separable convolutions. CoRR 2016, abs/1610.02357, 1610.02357.

16. Chollet, F. In Deep Learning with Python; Manning Publications Co: New York, U.S.A, 2018.

17. Kotikalapudi, R., contributors: keras-vis. GitHub 2017.

18. Selvaraju, R.R., Abhishek, D., Ramakrishna, V., Michael, C., Devi, P., Dhruv, B. Grad-cam: Why did you say that? visual explanations from deep networks via gradient-based localization. CoRR 2016, abs/1610.02391, 1610.02391.

19. Tobias, J. S., Alexey, D., Thomas, B., Martin, A. R. Striving for simplicity: The all convolutional net. CoRR 2014, abs/1412.6806, 1412.6806.

20. Sanya, R., Ernest, M. Mapping spatial housing patterns using Deep Neural Networks and remote sensing data. In Neural Information Processing Systems Conference 2018 Machine Learning for the Developing World, 2018.

21. Albert, A.T., Kaur, J., Gonzalez, M.C. Using convolutional networks and satellite imagery to identify patterns in urban environments at a large scale. ACM SigKDD 2017 Conference 2017.

22. Cheng, G., Han, J., Lu, X. Remote sensing image scene classification: Benchmark and state-of-the-art. In Proceedings of the IEEE 2017, 105(10), 1865-1883.

23. Jean, N., Burke, M., Xie, M., Davis, W. M., Lobell, D. B., Ermon, S. Combining satellite imagery and machine learning to predict poverty. Sciencemag 2016, 353(6301), 790-794.

24. Basu, S., Ganguly, S., Mukhopadhyay, S., DiBiano, R., Karki, M., Nemani, R. DeepSat-A learning framework for satellite imagery. arXiv:1509.03602v1 [cs.CV] 11 Sep 2015.

25. Deng, J., Dong, W., Socher, R., Li, L.-J., Li, K., Fei-Fei, L. Imagenet: A large-scale hierarchical image database. IEEE Int. Conf. Comput. Vis. Pattern Recognit 2009, 248-255.

26. Rosenblatt, F. The perceptron: A probabilistic model for information storage and organization in the brain. Psychological Review 1958, 65(6), 386-408. 
27. Moustris, K. P., Douros, K., Nastos, P. T., Larissi, J. K., Anthracopoulos, M. B., Paliatsos, A. G., Priftis, K. N. Seven days ahead forecasting of childhood asthma admissions using artificial neural networks in Athens, Greece. In International Journal of Environmental Research 2012, 22, 93-104.

28. Capinha, C., Gomes, E., Reis, E., Rocha, J., Sousa, C. A., Rosario, V. E., Almeida, A. P. Present habitat suitability for anopheles atroparvas (diptera, culicidae) and its coincidence with former malaria areas in mainland Portugal. Geospatial Health 2009, 3, 177-187.

29. Kiang, R., Adimi, F., Soika, V., Nigro, J., Singhasivanon, P., Sirichaisinthop, J., Leemingsawat, S., Apiwathnasorn, C., Looareesuwan, S. Metereological, environmental remote sensing and neural network analysis of epidemiology of malaria transmission in Thailand. Geospatial Health 2006, 1, 71-84.

30. Rajabi, M., Ali, M., Petter, P., Ahad, B. Environmental modelling of visceral leishmaniasis by susceptibility-mapping using neural networks: a case study in north-western Iran. Geospatial Health 2014, 9(1), 179-191.

31. Looney, C. Radial basis functional link nets and fuzzy reasoning. Neurocomputing 2002, 48, 489-509.

32. Facebook, L., Center for International Earth Science Information Network Columbia University. High Resolution Settlement Layer (HRSL). Source imagery for HRSL 2016 DigitalGlobe. 2016.

33. Tatem, A. J., Gething, P. W., Bhatt, S., Weiss, D., Pezzulo, C. Pilot high resolution poverty maps. 2013.

34. Alkire, S., Santos, M. Acute multidimensional poverty: A new index for developing countries. Technical report; UNDP-HDRO, New York, 2010.

35. Borremans, B., Jonas, R., Niel, H., and Herwig, L. The shape of the contact-density function matters when modelling parasite transmission in fluctuating populations. Royal Society Open Science 2017, 4, doi:10.1098/rsos.171308.

36. Hu, H., Karima, N., Philip, E. The scaling of contact rates with population density for the infectious disease models. Mathematical Biosciences 2013, 244(2013), 125-134. doi:10.1016/j.mbs.2013.04.013

37. Sanya, R., Mwebaze, E. Using socio-economic well-being to predict geospatial epidemic intensity in a developing country setting. In AGILE 20182018.

38. Robinson, C., Fred, H., Bistra, D. A deep learning approach for population estimation from satellite imagery. CoRR 2017, abs/1708.09086 1708.09086.

39. Baltrusaitis, T., Ahuja, C., Morency, L.-P. Multimodal Machine Learning: A Survey and Taxonomy. arXiv:1705.09406v2 [cs.LG] 2017.

40. Patterson, J. and Adam, G. In Deep Learning: A Practitioner's Approach; Loukides, M., McGovern, T., Eds.; O'Reilly Media Inc: Sebastopol, U.S.A, 2017.

41. Chollet, F., et al. Keras. GitHub 2015.

42. Abadi, M., Agarwal, A., Barham, P., Brevdo, E., Chen, Z., Citro, C., Corrado, G., Davis, A., Dean, J., Devin, M., Ghemawat, S., Goodfellow, I., Harp, A., Irving, G., Isard, M., Jia, Y., Kaiser, L., Kudlur, M., Levenberg, J., Man, D., Monga, R., Moore, S., Murray, D., Shlens, J., Steiner, B., Sutskever, I., Tucker, P., Vanhoucke, V., Vasudevan, V., Vinyals, O., Warden, P., Wicke, M., Yu, Y., Zheng, X. TensorFlow: Large-scale machine learning on heterogeneous distributed systems. arXiv preprint 2015, 1603.04467.

43. Smilkov, D., Nikhil, T., Been, K., Fernanda, B.V., Martin, W. Smoothgrad: removing noise by adding noise. CoRR 2017, abs/1706.03825. 1706.03825.

44. Karen, S., Andrea, V., Andrew, Z. Deep inside convolutional networks: Visualising image classification models and saliency maps. CoRR 2013, abs/1312.6034, 1312.6034.

Sample Availability: The three public datasets used and/or analyzed during the current study are available from the corresponding author on reasonable request or, they may be obtained directly from their respective websites for free. However, the epidemic data are available from the corresponding author subject to approval by the Ministry of Health, Government of the Republic of Uganda. 